\title{
THE PERFORMANCE OF SOUTH AFRICAN ENGLISH FIRST LANGUAGE CHILD SPEAKERS ON A "LOW LINGUISTICALLY LOADED"CENTRALAUDITORY PROCESSING TEST PROTOCOL
}

\author{
Nicole G. Campbell* and Wayne J. Wilson* \\ * Department of Communication Pathology, University of Pretoria, Pretoria, South Africa

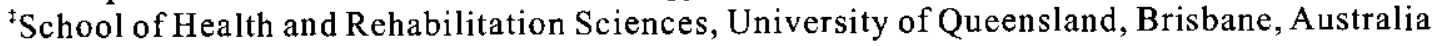

\begin{abstract}
The lack of standardized tests for central auditory processing disorders (CAPD) in South Africa (SA) led to the formation of a SA CAPD Taskforce, and the interim development of a" Low Linguistically Loaded i"CAPD test protocol using test recordings from the 'Tonal and Speech Materials for Auditory Perceptual Assessment Disc 2.0'. This study compared the performance of 50 SA English first language child speakers (aged 8 to 12 years of age) on this protocol, with the previously published American normative data of Bellis $(1996,2003)$. Results with respect to predicted pass criteria as calculated by mean-2SD cutoffs, suggested that the SA speakers performed of a lower level than the American speakers by an average of $5.3 \%$ per ear for the two pair dichotic digits test, $1.9 \mathrm{~dB}$ for the masking level difference test, $8.8 \%$ per ear for the frequency pattern test - humming report. $14.5 \%$ per ear for the frequency patterns test - verbal report, and $39.7 \%$ per ear for the low pass filtered speech test. Consequently, the Bellis (1996, 2003) data was not considered appropriate for immediate use as normative data in $S A$. Instead, the preliminary data provided in this study was recommended as interim normative data for SA English first language child speakers until larger scale SA normative data can be obtained.
\end{abstract}

\section{INTRODUCTION}

This study served as a direct follow-on from the Saleh, Campbell and Wilson (2003) study published on pages 19 to 25 of this edition of the South African Journal of Communication Disorders. Whereas the Saleh et al. (2003) study investigated the performance of South African (SA) English first and second language adult speakers on the "Low Linguistically Loaded" CAPD test protocol selected by the SA Central Auditory Processing Disorder (CAPD) Taskforce, the present study investigated the performance of SA English first language child speakers on selected tests, from the same test protocol.

\section{METHODOLOGY} AIMS

Following on from the SA CAPD Taskforce's proposed" Low Linguistically Loaded" interim CAPD test protocol, and the resulting need for SA specific normative data, this study used a comparative research design (Leedy \& Ormrod, 2001) to:

- Obtain preliminary normative data from SA English first language child speakers, on four tests of CAPD suitable for use in the proposed test protocol.

- Determine if the performances of the SA English speakers differed from the previously reported American normative data of Bellis (1996,2003).

\section{SUBJECTS}

Fifty child subjects ( 10 each from the $8,9,10$, 11 and 12 year old age groups) were conveniently sampled from four mainstream primary schools in the Gauteng region. The children were recruited by approaching the principals of the schools. Permission was obtained from the principals to send letters home with the children that outlined the aim of the study, the test procedures as well as a request for volunteers to participate in the study. The sample size for each age interval was based on the minimum sample size required for utilizing the Means Procedure of the SAS program (SAS Institute Inc., 1999) and the time limitations of the study. The subjects all spoke SA English as their first language (based on the first language leant at school, and the first language used in general day-to-day living) and were all of the same ethnic background, namely white English first language speakers. They had no developmental or learning disabilities, no known medical history of adverse neurological or medical conditions, and normal pure tone thresholds and acoustic immittance results (Hall \& Mueller, 1997; Martin \& Clark, 2000). All criteria, except the pure tone and acoustic immittance requirements, were confirmed by subject and parental report only.

\section{MATERIALS AND APPARATUS}

A subject information sheet and letter of consent were used to explain the purpose and nature of the study. A biographical questionnaire, a WelchAllyn 3.5 V HAL Otoset otoscope, an audiometer (Interacoustic AC30 audiometer with Telephonic TDH-50 earphones), an acoustic immittance meter (GSI 28A middle ear analyzer), a compact disk player (single disc Philips portable AX1000), and the CID W-1 list of spondees (presented live voice), were used to ensure the subjects had no history or peripheral hearing deficits that could adversely affect the CAPD testing. The same audiometer and compact disk player, and the 'Tonal and Speech Materials for Auditory Perceptual Assessment Disc 2.0' (Wilson \& Strouse, 1998), were used to obtain the CAPD test data.

Of all the tests available on the 'Tonal and 
Disc 2.0' (Wilson \& Strouse, 1998), only the following four tests were used: the two pair dichotic digits test (a low linguistically loaded dichotic speech test), the low-pass filtered speech test (a monaural low redundancy test), the frequency patterns test (a temporal patterning test), and the speech masking level difference test (a binaural interaction test). The two pair dichotic test was selected, as this task was more challenging than the use of single digits, yet simple enough for young children (Bellis, 1996, 2003). The remaining three tests were selected as the child age norms for these tests were better defined than for other tests of CAPD (Bellis, 1996, 2003). All tests were suitable for inclusion in the" low linguistically loaded"CAPD test protocol as recommended by the SA CAPD Taskforce (South Africa Central Auditory Processing Taskforce, 2000, 2001).

It must be noted that the low-pass filtered speech test recording used in this study was from the 'Tonal and Speech Materials for Auditory Perceptual Assessment, Disc 2.0' (Wilson \& Strouse, 1998). This recording used monosyllabic words from List 3 of the Northwestern University Auditory Test No. 6 (N. U. No. 6), spoken by a female, and low-pass filtered with a $1500 \mathrm{~Hz}$ cutoff at $115 \mathrm{~dB} /$ octave (Wilson, Zizz, Shanks \& Causey, 1990). The low-pass filtered speech test recording used by Bellis $(1996,2003)$ was from Auditec, St Louis. This recording used monosyllabic words from List 3 of the Northwestern University Auditory Test No. 6 (N. U. No. 6), spoken by a male, and low-pass filtered with a $1000 \mathrm{~Hz}$ cutoff (Bellis, 1996, 2003).

All testing was conducted in a sound-treated test booth and all audiometric equipment and test environments complied with the South African Bureau of Standards specifications.

\section{PROCEDURES}

On arrival for testing, the parents of each subject completed the informed consent and pre-test questionnaire forms, and each subject underwent otoscopic, pure tone, speech reception and acoustic immittance testing. Subjects who met the selection criteria were then tested on the selected CAPD tests from the 'Tonal and Speech Materials for Auditory Perceptual Assessment Disc 2.0' (Wilson \& Strouse, 1998). Both the order of test presentation, and the order of ear testing (where appropriate), were randomized.

The test procedures used were as per those described by Saleh et al. (2003) on pages 21 to 22 of this edition of the South African Journal of Communication Disorders, with the following modifications:

1) Each subject was familiarised with the test stimuli prior to the tests being applied. For the twopair dichotic digits, each subject was asked to repeat sequences of four digits spoken live voice by the examiner. For the low-pass filtered speech and speech masking level difference tests, the test-words were read to each subject by the examiner and their meaning discussed. For the frequency pattern test, each subject was asked to verbally label and hum patterns as hummed by the examiner. Each subject was also informed that the accent of the recorded speech material was American, and that the pronunciation of some of the words differed slightly from the SA pronunciation. Whilst similar familiarisation techniques were used by Bellis (1996, 2003 ), their use in the present study was primarily to counter the possible linguistic bias resulting from assessing SA English first language speaking children using CAPD test materials recorded in American English (Wilson \& Strouse, 1998).

2) Stimulus presentation levels were set to 50 $\mathrm{dBSL}$ relative to the average pure tone threshold at 0.5 , 1 and $2 \mathrm{kHz}$. These levels were different from that of Saleh et al. (2003) and Bellis' (1996, 2003) use of 50 dBSL relative to the $1000 \mathrm{~Hz}$ threshold, $50 \mathrm{dBSL}$ relative to spondee threshold, or $50 \mathrm{dBHL}$, depending on the test involved. Table 1 summarises the differences in presentation levels between this study and those used by Bellis $(1996,2003)$. The reason for the different levels was that the normative data being generated by this study was used in a subsequent doctoral study by Campbell (2003) where spondee thresholds were not measured.

3) The frequency pattern test was presented twice. For the first presentation, each subject had to say the patterns back. For the second presentation, each subject had to hum the patterns back. The same frequency patterns and protocol were used for each response format.

\section{DATA COLLECTIONAND ANALYSIS}

All subject responses were recorded manually and scored off-line. Group performances on each CAPD test were described using means and standard deviations. Comparisons with the normative data of Bellis $(1996,2003)$ were completed using mean-2SD cut-off values only. These mean $2 \mathrm{SD}$ values were used as they were the only values provided by Bellis $(1996,2003)$ (her report did not report sample sizes, subject selection criteria, or raw data).

\section{RESULTS AND DISCUSSION}

Table 2 shows the results obtained for the SA English first language child speakers, and the related Bellis (1996, 2003) American normative data, on the CAPD tests assessed. Compared to the American normative data (using the mean-2SD values only), the

Table 1. Differences in the test procedures employed by this study and by Bellis $(1996,2003)$.

\begin{tabular}{|c|c|c|}
\hline Test & $\begin{array}{l}\text { Audiometer settings used in } \\
\text { current study }\end{array}$ & $\begin{array}{c}\text { Audiometer settings used by Bellis } \\
(1996,2003)\end{array}$ \\
\hline $\begin{array}{l}\text { The two pair dichotic } \\
\text { digits test }\end{array}$ & & $\begin{array}{l}50 \text { dBSL relative to the spondee } \\
\text { threshold of the better ear }\end{array}$ \\
\hline $\begin{array}{l}\text { The frequency } \\
\text { patterns test }\end{array}$ & $\begin{array}{l}50 \text { dBSL relative to the average } \\
\text { pure tone threshold at } 500,1000 \\
\text { and } 2000 \mathrm{~Hz} \text { of the better ear }\end{array}$ & $\begin{array}{l}50 \mathrm{dBSL} \text { relative to the } 1000 \mathrm{~Hz} \\
\text { threshold of the test car }\end{array}$ \\
\hline $\begin{array}{l}\text { The low-pass filtered } \\
\text { speech test }\end{array}$ & & $50 \mathrm{dBHL}$ \\
\hline
\end{tabular}


Table 2: SA English first language speaking child data (mean \pm SD and mean - 2SD), and Bellis' (1996, 2003) American Normative data (mean - 2SD only, in bold)

\begin{tabular}{|c|c|c|c|c|c|c|c|c|c|c|}
\hline \multirow[t]{2}{*}{$\begin{array}{l}\text { Age in } \\
\text { years }\end{array}$} & \multirow{2}{*}{$\begin{array}{l}\text { Test } \\
\text { Ear }\end{array}$} & \multicolumn{2}{|c|}{ Dichotic digits test } & \multicolumn{2}{|c|}{$\begin{array}{c}\text { Frequency pattern test - } \\
\text { verbal }\end{array}$} & \multicolumn{2}{|c|}{$\begin{array}{l}\text { Frequency pattern test } \\
\text { Jhumming }\end{array}$} & \multicolumn{2}{|c|}{ Low-pass filtered speech } & \multirow{2}{*}{$\begin{array}{l}\text { Speech } \\
\text { MLD test } \\
\text { (dB) }\end{array}$} \\
\hline & & R (\%) & $L(\%)$ & $\mathbf{R}(\%)$ & $\mathbf{L}(\%)$ & R $(\%)$ & $\mathbf{L}(\%)$ & $\mathbf{R}(\%)$ & $L(\%)$ & \\
\hline $\begin{array}{c}8 \\
(n=10)\end{array}$ & $\begin{array}{c}\text { Mean } \pm \text { SD } \\
\text { Mean-2SD } \\
\text { Bellis }\end{array}$ & $\begin{array}{c}87.0 \pm 7.5 \\
71.9 \\
75 \\
\end{array}$ & $\begin{array}{c}77.3 \pm 8.8 \\
59.7 \\
65 \\
\end{array}$ & $\begin{array}{c}49.4 \pm 14.5 \\
20.4 \\
42\end{array}$ & $\begin{array}{c}50.2 \pm 12.9 \\
24.5 \\
42\end{array}$ & $\begin{array}{c}56.8 \pm 8.8 \\
39.2 \\
42\end{array}$ & $\begin{array}{c}56.4 \pm 9.7 \\
37.0 \\
42 \\
\end{array}$ & $\begin{array}{c}43.5 \pm 13.1 \\
17.2 \\
\mathbf{7 0} \\
\end{array}$ & $\begin{array}{c}37.5 \pm 15.5 \\
6.5 \\
70\end{array}$ & $\begin{array}{c}5.2 \pm 1.1 \\
2.9 \\
\mathbf{5 . 5}\end{array}$ \\
\hline $\begin{array}{c}9 \\
(n=10)\end{array}$ & $\begin{array}{c}\text { Mean } \pm \text { SD } \\
\text { Mean-2SD } \\
\text { Bellis }\end{array}$ & $\begin{array}{c}88.0 \pm 7.4 \\
73.1 \\
\mathbf{8 0}\end{array}$ & $\begin{array}{c}82.0 \pm 7.9 \\
66.2 \\
75\end{array}$ & $\begin{array}{c}64.0 \pm 9.6 \\
44.9 \\
63 \\
\end{array}$ & $\begin{array}{c}64.0 \pm 7.7 \\
49.1 \\
63 \\
\end{array}$ & $\begin{array}{c}67.2 \pm 6.2 \\
54.8 \\
\mathbf{6 3} \\
\end{array}$ & $\begin{array}{c}68.2 \pm 6.3 \\
55.6 \\
63\end{array}$ & $\begin{array}{c}49.5 \pm 9.3 \\
31.0 \\
68\end{array}$ & $\begin{array}{c}50.5 \pm 12.6 \\
25.4 \\
68\end{array}$ & $\begin{array}{c}5.8 \pm 0.8 \\
4.1 \\
5.5\end{array}$ \\
\hline $\begin{array}{c}10 \\
(n=10)\end{array}$ & $\begin{array}{c}\text { Mean } \pm \text { SD } \\
\text { Mean-2SD } \\
\text { Bellis }\end{array}$ & $\begin{array}{c}93.3 \pm 3.6 \\
86.2 \\
\mathbf{8 5}\end{array}$ & $\begin{array}{c}90.0 \pm 8.2 \\
73.7 \\
78\end{array}$ & $\begin{array}{c}73.6 \pm 8.5 \\
56.7 \\
78 \\
\end{array}$ & $\begin{array}{c}72.6 \pm 6.0 \\
60.7 \\
78 \\
\end{array}$ & $\begin{array}{c}77.0 \pm 4.3 \\
69.3 \\
\mathbf{7 8}\end{array}$ & $\begin{array}{c}75.8 \pm 5.2 \\
65.4 \\
78\end{array}$ & $\begin{array}{c}52.5 \pm 11.6 \\
29.3 \\
72\end{array}$ & $\begin{array}{c}54.0 \pm 8.8 \\
36.5 \\
\mathbf{7 2}\end{array}$ & $\begin{array}{c}5.4 \pm 0.9 \\
3.7 \\
5.5\end{array}$ \\
\hline $\begin{array}{c}11 \\
(n=10)\end{array}$ & $\begin{array}{c}\text { Mean } \pm \text { SD } \\
\text { Mean-2SD } \\
\text { Bellis }\end{array}$ & $\begin{array}{c}94.3 \pm 6.1 \\
82.0 \\
90\end{array}$ & $\begin{array}{c}92.0 \pm 5.1 \\
82,0 \\
\mathbf{8 8}\end{array}$ & $\begin{array}{c}80.0 \pm 4.6 \\
81.8 \\
78 \\
\end{array}$ & $\begin{array}{c}81.2 \pm 5.7 \\
70.8 \\
78 \\
\end{array}$ & $\begin{array}{c}82.4 \pm 5.7 \\
69.9 \\
78\end{array}$ & $\begin{array}{c}82.8 \pm 4.6 \\
71.0 \\
78\end{array}$ & $\begin{array}{c}57.0 \pm 9.5 \\
38.0 \\
\mathbf{7 5} \\
\end{array}$ & $\begin{array}{c}55.0 \pm 8.2 \\
38.0 \\
75\end{array}$ & $\begin{array}{c}5.8 \pm 1.7 \\
2.4 \\
5.5\end{array}$ \\
\hline $\begin{array}{c}12 \\
(n=10)\end{array}$ & $\begin{array}{c}\text { Mean } \pm \text { SD } \\
\text { Mean-2SD } \\
\text { Bellis }\end{array}$ & $\begin{array}{c}93.5 \pm 4.6 \\
84.3 \\
90\end{array}$ & $\begin{array}{c}92.8 \pm 5.1 \\
82.6 \\
90\end{array}$ & $\begin{array}{c}82.4 \pm 10.7 \\
61.0 \\
80 \\
\end{array}$ & $\begin{array}{c}79.6 \pm 11.4 \\
56.8 \\
\mathbf{8 0}\end{array}$ & $\begin{array}{c}84.8 \pm 9.2 \\
66.4 \\
\mathbf{8 0}\end{array}$ & $\begin{array}{c}82.4 \pm 8.3 \\
65.9 \\
\mathbf{8 0}\end{array}$ & $\begin{array}{c}69.0 \pm 7.8 \\
53.5 \\
78 \\
\end{array}$ & $\begin{array}{c}67.9 \pm 7.1 \\
53.8 \\
\mathbf{7 8} \\
\end{array}$ & $\begin{array}{c}6.2 \pm 1.2 \\
3.7 \\
\mathbf{5 . 5}\end{array}$ \\
\hline
\end{tabular}

\section{R - Right}

L - Left

than Bellis' (1996, 2003) American normative data for all tests except the right ear score of the 10 year olds on the two pair dichotic digits test.

Ranking the mean-2SD scores of the SA English first language child speakers showed that they performed most like their American English speaking counterparts on the two pair dichotic digits test (averaging 5.3\% lower/ear), followed by the specch masking level difference test (averaging 1.9 $\mathrm{dB}$ lower), the frequency pattern test - humming report (averaging 8.8\% lower/ear), the frequency patterns test - verbal report (averaging 14.5\% lower/ear), and lastly the low-pass filtered speech test (averaging 39.7\% lower/ear).

The most likely factor to have influenced the two pair dichotic digits, the low pass filtered speech, and the speech masking level differencc test scores, was the American English accent of the test recordings. Whilst these tests were chosen because of thíeir relatively low linguistic load, there was still sufficient load to disadvantage the SA English first language child speakers.

An accent mismatch was not enough, however, to explain the much poorer mean-2SD scores obtained by the SA English first language child speakers on the low-pass filtered speech test. This effect was more likely to have resulted from the different $C D$ recordings used by this study and those of Bcllis $(1996,2003)$. It is possible that the words on this study's 'Tonal and Speech Materials for Auditory Perceptual Assessment, Disc 2.0' (Wilson \& Strouse, 1998) recording may harder to recognise (Bellis, 1996, 2003). The lower scores were of particular concern when considering the very low $(<20 \%)$ scores obtained by the 8 year old group, and the generally low scores $(<40 \%)$ obtained by the 9 to 11 year old groups. Overall, these results suggest that the 'Tonal and Speech Materials for Auditory Perceptual Asscssment, Disc 2.0' (Wilson \& Strouse, 1998) recording of the low-pass filtered speech test should be approached with caution when assessing SA English first language child speakers.
Similarly, an accent mismatch also cannot explain the lower mean-2SD scores obtained by the SA English first language subjects on the frequency patterns test on both the verbal and humming report. Further research is needed to identify the reasons for these differences, although the better scores obtained on the humming report suggest a possible influence of the maturity level of the corpus callosum (Bellis, 2003). Similar to the performance on the low-pass filtered speech test, the very poor scores obtained by the 8 year old group on the frequency patterns test for verbal report, suggest that the frequency patterns test on the 'Tonal and Speech Materials for Auditory Perceptual Assessment Disc 2.0's' (Wilson \& Strouse, 1998) should also be used with caution in this younger age group.

\section{CONCLUSIONS}

Preliminary normative data was obtained for SA English first language child speakers on four tests of CAPD selected from the 'Tonal and Speech Materials for Auditory Perceptual Assessment Disc 2.0' (Wilson \& Strouse, 1998). Each test was chosen because of its suitability for use in the" low linguistically loaded"test protocol proposed by the SA CAPD Taskforce.

On average, the SA English first language child speakers pcrformed worse than the Bellis $(1996,2003)$ American data (as calculated by comparing mean2SD cutoffs). As a result, the Bellis (1996, 2003) American normative data was not considered appropriate for immediate use in SA. Instead, the SA data provided in this study was recommended for use as preliminary normative data for SA English first language child speakers, until larger scale SA normative data can be obtained. In particular, the subjects lower scores on the low-pass filtered speech test contained on the 'Tonal and Speech Materials for Auditory Perceptual Assessment Disc 2.0' (Wilson, \& Strouse, 1998), suggest that this test should be approached with caution when assessing South 
Limitations of this study are noted and the results cannot be generalized beyond the subject, stimulus and recording parameters used.

Note: The 'Tonal and Speech Materials for Auditory Perceptual Assessment Disc 2.0' (Wilson, \& Strouse, 1998) is available from Professor Richard Wilson PhD, Audiology (126), VA Medical Centre, Mountain Home, Tennessee 37684, ph +1423926 1171 ext 7553 , fax +14232326903 , email Richard.Wilson $2($ m ed.va.gov or wilson.richard@mtn-home.va.gov. Professor Wilson does not charge a formal price for the $C D$, as it was produced by USA Veterans Affairs. He has requested that you send a "donation (postal order)" of US\$50100 to his research fund - the East Tennessee State University (ETSU) Foundation.

\section{REFERENCES}

Bellis, T. J. (1996). Assessment and management of central auditory processing disorders in the educational setting. From science to practice. San Diego: Singular Publishing Group.

Bellis, T. J. (2003). Assessment and management of central auditory processing disorders in the educational setting: From science to practice (2nd ed.). San Diego: Singular Publishing Group.

Campbell, N. G. (2003). The Central Auditory Processing and Continuous Performance of children with Attention Deficit Hyperactivity Disorder (ADHD) in the medicated and nonmedicated state. Unpublished Doctoral
Dissertation. University of Pretoria, Pretoria.

Hall, J. W., \& Mueller, H. W. (1997). Audiologists' desk reference. San Diego: Singular Publishing Group.

Leedy, P. D., \& Ormrod, J. E. (2001). Practical Research: Planning and Design (7th ed.). Upper Saddle River, New Jersey: Merrill Prentice Hall.

Martin F. N., \& Clark, J. G. (2000). Introduction to audiology (7th ed.). Boston: Allyn and Bacon.

SAS Institute Inc. (1999). SAS/STAT. User's Guide Version 8. Cary, NC: SA SInstitute Inc.

Saleh, S., Campbell, N. G., \& Wilson, W. J. (2003). The performance of South African English first and second language adult speakers on a" low linguistically loaded"central auditory processing test protocol. South African Journal of Communication Disorders, 50, 19-25.

South Africa Central Auditory Processing Disorder Taskforce. (2000). SA CAPD taskforce business plan. Unpublished Document. Pretoria, South A frica.

South Africa Central Auditory Processing Disorder Taskforce. (2001). SA CAPD taskforce: $A n$ update. Unpublished Document. Pretoria, South Africa.

Wilson, R.H., \& Strouse, A. (1998). Tonal and speech materials for auditory perceptual assessment disc 2.0. Tennessee: Department of Veterans Affairs.

Wilson, R. H., Zizz, C. A., \& Sperry, J. L. (1994). Masking level difference for spondaic words in 2000 -msec bursts of broadband noise. Journal of 